\title{
El cambio climático en el
}

\section{bachillerato. Aportes pedagógicos para su abordaje}

\section{Climate change in high school. Pedagogical contributions to its approach}

\author{
Laura O. Bello Benavides \\ Instituto de Investigaciones \\ en Educación, Universidad \\ Veracruzana, México \\ labello@uv.mx,
}

\author{
Gloria E. Cruz Sánchez \\ Instituto de Investigaciones \\ en Educación, Universidad \\ Veracruzana, México \\ gcruz@uv.mx
}

\author{
Pablo Á. Meira Cartea \\ Faculta de Ciencias da \\ Educación, Departamento \\ de Pedagoxía e Didáctica, \\ Universidad de Santiago de \\ Compostela, España \\ pablo.meira@usc.es
}

\author{
Édgar J. González Gaudiano \\ Instituto de Investigaciones \\ en Educación, Universidad \\ Veracruzana, México \\ edgagonzalez@uv.mx
}

RESUMEN • La investigación presentada tiene como propósito conocer elementos de las representaciones sociales (RS) sobre cambio climático (CC) en estudiantes de bachillerato general y tecnológico del estado de Veracruz, y formular aportaciones pedagógicas orientadas a promover acciones ecociudadanas en estudiantes de este nivel. Se ha realizado una investigación cuantitativa con una muestra de 858 estudiantes, a través de un cuestionario demoscópico conformado por 43 preguntas cerradas. La hipótesis de investigación es: si no existe diferencia significativa entre las RS sobre CC en los dos grupos de estudio, cabe pensar que es la cultura común la que configura en mayor medida dicha RS. Los resultados muestran que este colectivo construye una RS del CC limitada a su dimensión biofísica con presencia de conceptos difusos, sin identificarse diferencias significativas entre las RS de los grupos.

PALABRAS CLAVE: Cambio climático; Educación ambiental; Representaciones sociales; Bachillerato.

ABSTRACT - This research aims to know the elements of the social representations (SR) on climate change (CC) in students of general (BG) and technological (BT) high school of the state of Veracruz, and make pedagogical contributions oriented to promote eco-citizen actions in students of this level. A quantitative investigation was carried out with a sample of 858 students, through a demographic questionnaire of 43 closed questions. The research hypothesis is: if there is no significant difference between the SRs on CC in the two study groups, it is possible to think that it is the common culture that configures this SR to a greater extent. The results show that this group builds a SR of the CC limited to its biophysical dimension with the presence of diffuse concepts, without identifying significant differences between the SRs of the groups.

KEY WORDS: Climate change; Environmental education; Social representations; High school.

1. Este artículo es parte del Proyecto Resclima, financiado por el Ministerio de Economía y Competitividad del Gobierno de España, en el marco del VI Programa Nacional 2016-18, convocatoria 2015, REF. EDU2015-63572-P y de la convocatoria de Proyectos I+D+i de Retos Investigación 2018, REF. RTI2018-094074-B-I00.

Recepción: julio 2019 • Aceptación: enero 2020 • Publicación: marzo 2021 


\section{INTRODUCCIÓN}

El CC se configura actualmente como el problema socioambiental de mayor complejidad debido a sus múltiples impactos a nivel ecológico, económico y social, además de la incertidumbre inherente a los sistemas climáticos (IPCC, 2014). También está la urgente necesidad de implementar acciones de mitigación y adaptación desde la esfera global hasta la regional y local.

Se han realizado múltiples estudios, desde el campo de la educación, sobre cómo la población representa y comprende el fenómeno (Allen y Crowley, 2017; González y Maldonado, 2013; Lorenzoni y Pidgeon, 2005; Meira, 2011; OECD, 2009). Otras investigaciones acerca de cómo abordar el CC desde el currículo formal apuntan a la necesidad de transitar hacia enfoques basados en la complejidad y con una orientación social y de participación comunitaria través de aprendizajes socioculturales (Allen y Crowley, 2017; Bangay y Blum, 2010; Stevenson, Nicholls y Whitehouse, 2017). Stapleton y Sezen-Barrie (2017), ponen de relieve el rol docente en los procesos educativos acerca del CC. En el campo de la Educación Ambiental han emergido propuestas pedagógicas y didácticas para la sensibilización y acción frente al CC (Bello, 2017; Meira, 2011; O’Neill y Hulme, 2009; Sauvé 2014). Pese a la diversidad de investigaciones, aún quedan muchas interrogantes por responder: ¿cómo incide el currículo en la representación del CC? ¿De qué manera el conocimiento/desconocimiento del CC influye en las acciones de respuesta?

La investigación aquí presentada se inscribe en un proyecto internacional de investigación educativa, RESCLIMA, que en su tercera oleada de acercamiento a las RS del CC, centra su atención en cómo los estudiantes de educación secundaria y bachillerato, entre 15 y 17 ańos, se apropian de la ciencia del CC -a través del currículum y de otras formas de difusión y propagación de las representaciones científicas-y la integran en sus representaciones sociales acerca del fenómeno. El estudio fue realizado con muestras de adolescentes de cinco países: Brasil, España, Italia, México y Portugal durante los años 2017 y 2018.

La muestra que corresponde a México se realizó en el estado de Veracruz, en escuelas de Bachillerato general (BG) y tecnológico (BT), cuyo propósito, además de contribuir al referido proyecto internacional, fue aportar elementos empíricos para formular estrategias educativas que articulen los programas de estudio vigentes en este nivel educativo, para contribuir a generar actitudes ecociudadanas frente a la crisis climática (Bello, 2017; Sauvé, 2014). Este documento ofrece los resultados de dicho estudio y expone algunas de las propuestas generadas.

\section{EL CAMBIO CLIMÁTICO EN EL ACTUAL MODELO EDUCATIVO DE BACHILLERATO}

El bachillerato en el sistema educativo mexicano se cursa en tres ciclos escolares y acoge a estudiantes de entre 15 y 18 ańos. Existen dos modalidades: BG y BT. El primero se distingue por ser bivalente, esto es el egresado, además de obtener el certificado de Bachillerato, accede al título de alguna carrera técnica que oferta la secretaría de Educación Pública (SEP, 2017).

La EA se ha incorporado en el bachillerato con el enfoque de Ciencia, Tecnología, Sociedad y Ambiente (CTSA) (Fernández, Pries y Villamañán, 2014), con una propuesta más transversal y transdisciplinaria, donde el CC tiene más posibilidades de estar integrado. Este enfoque se articula con una visión de la educación escolar centrada en el aprendizaje de los alumnos y en el desarrollo de competencias educativas. Base pedagógica desde la que se diseñan los programas de estudio (SEP, 2017) que, en su conjunto, van a constituir la propuesta curricular. 
El abordaje del CC mediante el enfoque de CTSA se formula a través del conocimiento de sus causas y se plantean algunas acciones, fundamentalmente de mitigación (SEP, 2017). Para que esta orientación se pueda concretar es preciso realizar estudios acerca de cómo el currículum está incidiendo en las prácticas curriculares (Carr, 1997) y en los conocimientos y representaciones del alumnado acerca del CC. Los resultados de estos estudios pueden contribuir al debate teórico-metodológico en este campo educativo, así como formular estrategias educativas apropiadas para inducir acciones efectivas, tanto de mitigación como de adaptación al CC, por parte del alumnado y, por extensión, de las familias y comunidades de referencia.

Es justo en este ámbito en el que esta investigación justifica su pertinencia: estudiar la configuración de las RS sobre el CC en estudiantes de bachillerato para definir estrategias de educación ambiental que les permitan valorar la relevancia y la significación del CC en sus vidas, así como para impulsar acciones efectivas en los planos individual y colectivo en materia de mitigación y adaptación.

Como marco interpretativo de los datos obtenidos en este estudio, se ha recurrido a la teoría de las RS de Moscovici (1979). Las RS son una modalidad de pensamiento práctico, de conocimiento de sentido común, cuyo propósito es la comunicación y la interpretación del contexto social. Este pensamiento práctico o de «sentido común» orienta la actuación de las personas y, a través de la comunicación, se convierte en un elemento de constitución de un universo mental socialmente consensuado. Los conocimientos que las nutren se presentan organizados y jerarquizados, además de estar caracterizados socialmente en función del contexto en el que emerge una RS concreta, así como de las características y tipo de comunicación que se presenta (Ibáñez, 1994).

Las RS están conformadas por tres dimensiones: la información, la representación y la actitud. La dimensión de la información remite a los contenidos de la representación y a su organización, que transita desde una organización difusa hasta una estructurada (Moscovici, 1979). La dimensión representacional remite a la imagen estilizada del objeto representado; es decir, a los elementos que lo configuran y su jerarquía (Jodelet, 2008). La actitud se hace evidente a través de la disposición a actuar por parte del sujeto frente al objeto representado, lo que define su posición valorativa respecto a este.

El estudio de la dimensión social de CC a través de la teoría de las RS es pertinente por dos razones principales:

1. Por la incorporación de conocimiento científico y su vulgarización a través de procesos de difusión y propagación, un fenómeno cada vez más frecuente y transcendente en las sociedades contemporáneas (Moscovici, 1979; Wagner y Hayes, 2011). El CC emerge como una noción -una representación científica- creada por las ciencias del clima, que ha generado conceptos e imágenes que dan cuenta de un conjunto de fenómenos reales y que se han propagado a la población hasta entrar a formar parte de la cultura popular.

2. Porque los conocimientos científicos no se suman entre sí y conservan el mismo significado en su periplo social, sino que se integran con conceptos e ideas de sentido común para interpretar un objeto social nuevo como el CC (Jodelet, 2008). Estos estudios, por tanto, permiten indagar cómo la ciencia se integra y representa en el marco de la cultura común, como está ocurriendo con la construcción del CC como «objeto social».

\section{DISEÑO METODOLÓGICO E INSTRUMENTO}

Los supuestos hipotéticos que han guiado el estudio son: 1) que la constitución de RS sobre CC está mediada por la integración de la cultura común y la científica, si bien tienden a situarse en el centro de las teorías profanas; y 2) que el currículo diferenciado del BT y BG podría incidir en la representación generando algunas diferencias en la RS entre los dos grupos de estudio. 
Para concretar los objetivos expresados se ha diseñado una ruta metodológica de corte cuantitativo. Se empleó un cuestionario conformado por 43 preguntas cerradas que exploran las tres dimensiones de las RS del CC. 32 ítems son tipo Likert, con cuatro opciones de respuesta, y el resto son cerradas con variado número de opciones de respuesta. El cuestionario ha sido elaborado por el grupo RESCLIMA. Se aclara que el instrumento es de tercera generación, esto es, ha sido empleado ya en estudios previos (Meira, Gutiérrez, Arto y Escoz, 2018).

Para el análisis de los datos obtenidos se plantearon siete categorías analíticas, teniendo en cuenta las dimensiones de la RS, las causas, las consecuencias y el propio concepto del CC (véase el cuadro 1). Las preguntas que no aparecen en el cuadro según un orden numérico progresivo corresponden a una categoría analítica adicional relacionada con la autovaloración de la información que tienen los estudiantes sobre el CC, que para los fines de este artículo no ha sido incluida.

Cuadro 1.

Categorías analíticas y preguntas

\begin{tabular}{|l|l|}
\hline \multicolumn{1}{|c|}{ Categoría analítica } & \multicolumn{1}{c|}{ Número de preguntas } \\
\hline 1. Información-efecto invernadero/GEI & $1,5,8,9,12,19,23,24$ \\
\hline 2. Información-zonas oscuras/creencias profanas & $4,6,7,14,15,16,18,22,27,31$ \\
\hline 3. Información-consecuencias & $2,3,10,20,21,25,30$ \\
\hline 4. Información-causas & $11,13,17$ \\
\hline 5. Información sobre CC & 28,29 \\
\hline 6. Acciones & $26,32,43$ \\
\hline 7. Representación del CC & 38,39 \\
\hline
\end{tabular}

\section{MUESTRA}

La población de estudio estuvo constituida por un total de 858 estudiantes de entre 15 y 17 años de BT y BG. Se buscó que la población fuera urbana en todos los casos, a fin de homogeneizar las condiciones poblacionales con el resto de los países participantes en el estudio. Para construir una muestra representativa, se segmentó la población veracruzana en cinco regiones: Norte, Centro 1, Centro 2, Puerto y Sur. La muestra quedó conformada por 379 estudiantes de BT y 479 de BG, con un error admitido de 5,0 \% y un margen de confianza de 95,0\%; de este modo se garantiza la representatividad de los datos.

\section{ANÁLISIS DE LOS DATOS}

El tratamiento analítico de los datos ha sido de tipo estadístico, previa captura y codificación en el software SPSS. La primera parte del tratamiento estadístico consistió en la elaboración de datos descriptivos que dieran cuenta del grado de conocimiento acerca de cada categoría analítica. Posteriormente, se realizó el tratamiento estadístico siguiente: Alfa de Cronbach para valorar la fiabilidad de cada una de las categorías.

Las diferencias significativas entre los dos grupos de estudio se determinaron mediante análisis de varianza (ANOVA) de una vía y con el estadístico $X^{2}$. Se aplicó el ANOVA para las categorías información-zonas oscuras/creencias profanas, información-consecuencias e información efecto invernadero/ GEI, debido al número de preguntas que se orientan a estas categorías y por tener la misma estructura. 
El grado de significación fue p 0,05. Para profundizar en la comparación de medias significativas entre los dos grupos se hizo una segunda prueba post hoc de Tukey, realizándose una comparación múltiple entre las preguntas de cada categoría. El estadístico $X^{2}$ se empleó para el resto de las categorías analíticas. La correlación de Pearson se usó para verificar si existe correlación entre las preguntas de cada categoría analítica y entre estas. El nivel de significancia bilateral empleado fue de 0,05 y 0,01.

\section{EL CAMBIO CLIMÁTICO DESDE LA MIRADA DE LOS ESTUDIANTES}

Se exponen los resultados y el análisis de los datos obtenidos por cada categoría analítica.

\section{Información-Efecto invernadero/GEI}

El Alfa de Cronbach para este bloque de preguntas fue de 0,937 , lo que indica una alta confiabilidad en las respuestas realizadas. La gráfica 1 muestra una comparación de medias entre los resultados obtenidos por los dos grupos de estudio: BG y BT. Se expresa a través del gráfico de cajas y bigotes que señalan los cuatro cuartiles que segmentan la frecuencia de cada opción de respuesta: no contestó (NC), totalmente verdadero (TV), probablemente verdadero (PV), probablemente falso (PF) y totalmente falso (TF). También se incluye el valor promedio (media) por grupo en cada respuesta, que se indica con el símbolo +. Para el cálculo de medias se otorgó el siguiente puntaje a las opciones de respuesta: dado que la respuesta correcta para todas las preguntas de esta categoría analítica es TV, su valor es 4, PV tuvo un valor de 3 , PF de 2 y TF de 1.

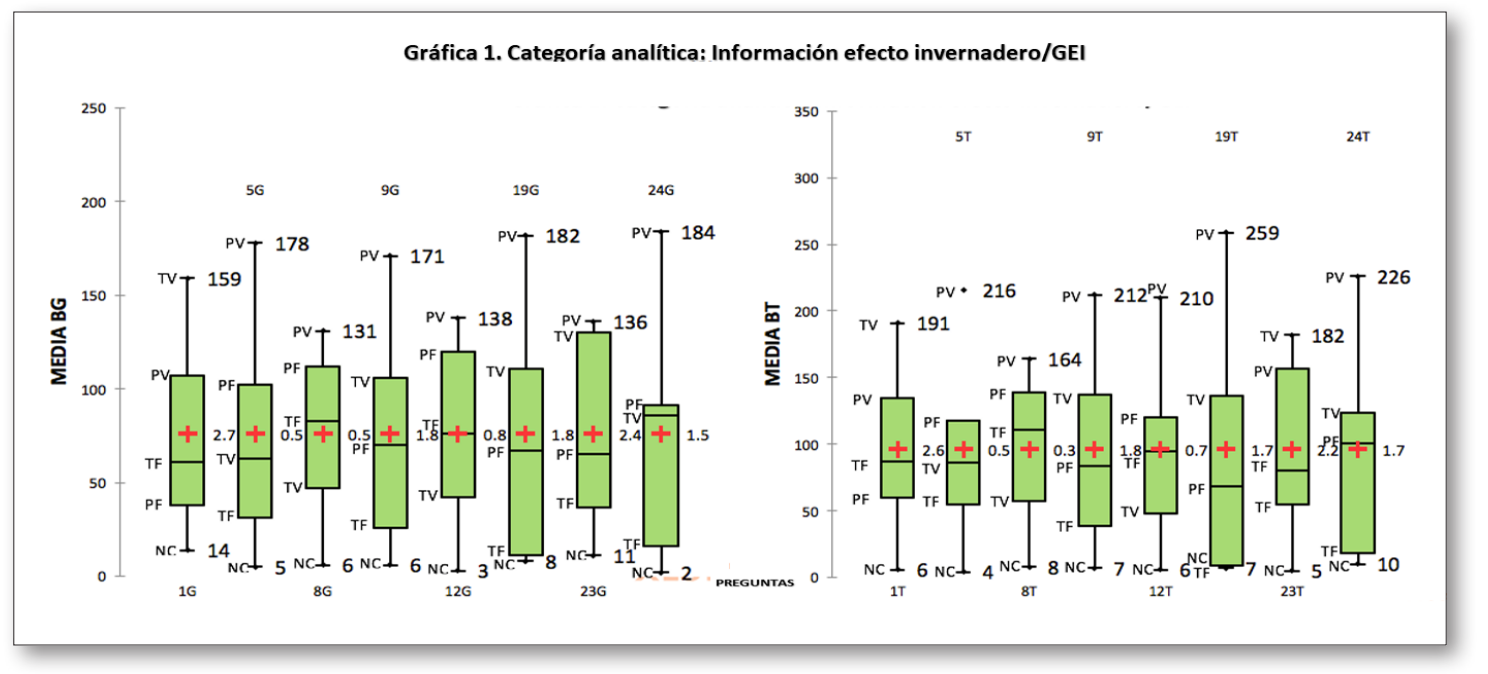

La exposición gráfica del peso de cada opción de respuesta (PV, TV, PF, TF) está esquematizado por la distribución de las dos cajas y los dos bigotes. Los bigotes (alambres), se ubican en los extremos inferior y superior de cada una de las cajas de las respuestas graficadas. El cuartil de menor frecuencia, expuesto en la parte inferior del gráfico, hace referencia a la opción «no contestó», en todos los casos; en la parte superior se expone la opción de mayor frecuencia, con su respectivo bigote. Las dos cajas representan los cuartiles dos y tres, que corresponden al resto de frecuencias de respuesta. Se muestra para cada ítem el valor de la opción de respuesta con menor y mayor frecuencia.

La media global y por grupo de estudio: en las respuestas a esta categoría analítica el BT y el BG se ubican por debajo del valor 2,5, que correspondería a un $50 \%$ de respuestas correctas por grupo. 
Como se observa en el gráfico 1 y en el cuadro 2 las preguntas 1 y 23 fueron las que sumaron mayor frecuencia de respuestas correctas: «TV», para los dos grupos de estudio. Para el resto de las preguntas, la opción «PV» fue la de mayor frecuencia. Este sesgo indica conocimientos precarios en relación con la dinámica del efecto invernadero y los GEI. Los datos muestran variabilidad entre las medias de cada respuesta en ambos grupos: BG y BT; esto es, el dominio de algunos conceptos, como «efecto invernadero" $\mathrm{y}$ "GEI», y carencia de conocimientos sobre sus efectos y sobre su papel en la vida planetaria. Estos datos revelan que el conocimiento que el alumnado posee acerca del efecto invernadero y los GEI es superficial y con escasos andamiajes consistentes con las ciencias del clima. Cabe destacar, además, la dificultad para asociar el efecto invernadero con las formas de vida en el planeta Tierra.

\section{Cuadro 2.}

Valoración de diferencias significativas entre BT y BG. Categoría: Efecto Invernadero/GEI

\begin{tabular}{|c|c|c|c|c|c|}
\hline Pregunta & $\begin{array}{c}\text { Media } \\
B G\end{array}$ & $\begin{array}{c}\text { Media } \\
B T\end{array}$ & $\begin{array}{l}\text { Media } \\
\text { Global }\end{array}$ & $\begin{array}{l}\text { Valor de } \\
\text { significancia } \\
(T U K E Y)\end{array}$ & $\begin{array}{l}\text { Frecuencia rpta. } \\
\text { (\%) Totalmente } \\
\text { verdadero }\end{array}$ \\
\hline 1. El efecto invernadero es un fenómeno natural & 2,7 & 2,6 & 2,6 & - & 40,8 \\
\hline $\begin{array}{l}\text { 5. Si dejamos de emitir gases de efecto inverna- } \\
\text { dero no nos afectará el CC }\end{array}$ & 0,5 & 0,5 & 0,5 & & 17,4 \\
\hline $\begin{array}{l}\text { 8. La mayor parte de los gases de efecto inverna- } \\
\text { dero presentes en la atmósfera provienen de } \\
\text { fuentes naturales }\end{array}$ & 0,5 & 0,3 & 0,3 & 0,323 & 12,1 \\
\hline 9. El $\mathrm{CO}_{2}$ es el principal gas responsable del CC & 1,8 & 1,8 & 1,8 & 0,620 & 28,3 \\
\hline $\begin{array}{l}\text { 12. De no ser por el efecto invernadero no existi- } \\
\text { ría la vida tal y como la conocemos }\end{array}$ & 0,8 & 0,7 & 0,8 & 0,550 & 10,5 \\
\hline $\begin{array}{l}\text { 19. El efecto invernadero se produce cuando los } \\
\text { gases retienen parte de la radiación reflejada } \\
\text { por la superficie terrestre }\end{array}$ & 1,8 & 1,7 & 1,8 & 0,851 & 28,8 \\
\hline $\begin{array}{l}\text { 23. El } \mathrm{CO}_{2} \text { es un componente natural de la at- } \\
\text { mósfera }\end{array}$ & 2,4 & 2,2 & 2,3 & 0,996 & 36,4 \\
\hline $\begin{array}{l}\text { 24. Si dejamos de emitir gases de efecto inverna- } \\
\text { dero seremos menos vulnerables al CC }\end{array}$ & 1,5 & 1,7 & 1,6 & 0,116 & 24,5 \\
\hline Media de la categoría analítica & 1,50 & 1,43 & 1,46 & & \\
\hline
\end{tabular}

La valoración de diferencias significativas entre los dos grupos de estudio se realizó a través de las pruebas ANOVA y Tukey; el factor de cruce fue la pregunta 1 (véase el cuadro 1). Los valores indican que no existen diferencias significativas en las respuestas a esta categoría entre los dos grupos de estudio, siendo todos mayores a P >0,05. Esto es, las diferencias curriculares entre el BG y BT no parecen incidir en el campo de la información acerca del efecto invernadero y los GEI.

El estudio de correlaciones para todas las categorías analíticas tuvo como propósito indagar qué información sobre el CC es la que están asociando, así como a qué datos la vinculan los estudiantes. La correlación en esta categoría analítica se examinó a través del estadístico de Pearson, con un nivel de significancia bilateral de $\mathrm{P}>0,05$ y $\mathrm{P}>0,01$. Los resultados señalan que existe correlación significativa de la pregunta 9 con las preguntas $19(\mathrm{P}=0,990), 23(\mathrm{P}=0,919)$ y $24(\mathrm{P}=0,981)$; además de la pregunta 1 con la $23(\mathrm{P}=0,928)$ y la pregunta 19 con la $24(\mathrm{P}=0,980)$. Estos datos revelan que el conocimiento acerca del principal GEI: $\mathrm{CO}_{2}$ (pregunta 9) se vincula con el efecto invernadero, con la composición de la atmósfera y con el CC. Esto es, existe claridad en vincular el efecto invernadero con este gas. Sin embargo, el efecto invernadero (pregunta 15) y las acciones de mitigación no se relacionan 
con el $\mathrm{CO}_{2}$ antrópico. Para el resto de los ítems la prueba no revela que los estudiantes de los grupos de investigación correlacionen información relativa a esta categoría analítica. En su conjunto, estos datos dan cuenta de que la información explorada en esta categoría presenta poca articulación entre sí.

La correlación con otras categorías analíticas se advierte en pocos casos. Para las preguntas que exploran las zonas oscuras/creencias profanas, se verifica únicamente asociación entre la 5 y la 18 ( $\mathrm{P}=$ 0,976). Esto es, con la creencia acerca de que el CC es resultado de la variabilidad climática natural. El mayor número de correlaciones se registran en la categoría analítica «consecuencias». Ambos grupos de estudio relacionan el $\mathrm{CO}_{2}$ antrópico (pregunta 9) con los impactos en enfermedades tropicales, con eventos extremos, con incremento del nivel del mar y con la desertificación, a través de las preguntas: $2(\mathrm{P}=0,880), 20(\mathrm{P}=0,906), 21(\mathrm{P}=0,911)$ y $25(\mathrm{P}=0,967)$.

En cuanto a la correlación entre datos de esta categoría con la de causas del CC, la prueba de Pearson no muestra correlación con el consumo de carnes rojas (pregunta 11), pero sí con el $\mathrm{CO}_{2}$ antrópico y el efecto invernadero $(\mathrm{P}=0,957)$. Destaca la correlación entre el $\mathrm{CO}_{2}$ antrópico y algunas acciones de respuesta como la reforestación $(\mathrm{P}=0,949)$. Estos datos revelan una correlación heterogénea entre la información sobre GEI y efecto invernadero con las causas y consecuencias del CC, y en algunos casos contradictoria, sobre todo en lo relacionado con la reducción de GEI antrópico y las consecuencias del CC.

\section{Información-zonas oscuras/creencias profanas}

Esta categoría analítica explora: 1) conocimientos sobre problemas ambientales que la población suele asociar con el CC, pero que la ciencia muestra que no tienen relación directa con él, como el agujero de la capa de ozono y la lluvia ácida (Bello, Meira y González, 2017; Meira, 2011; González y Maldonado, 2013); y 2) las creencias profanas acerca del CC. El Alfa de Cronbach para este bloque de preguntas fue de 0,990 , el valor de confiabilidad más alto en relación con el resto de las categorías analíticas. La gráfica 2 muestra el análisis estadístico realizado sobre la comparación de medias entre los resultados obtenidos en cada ítem de esta categoría analítica de ambos grupos: BT y BG.

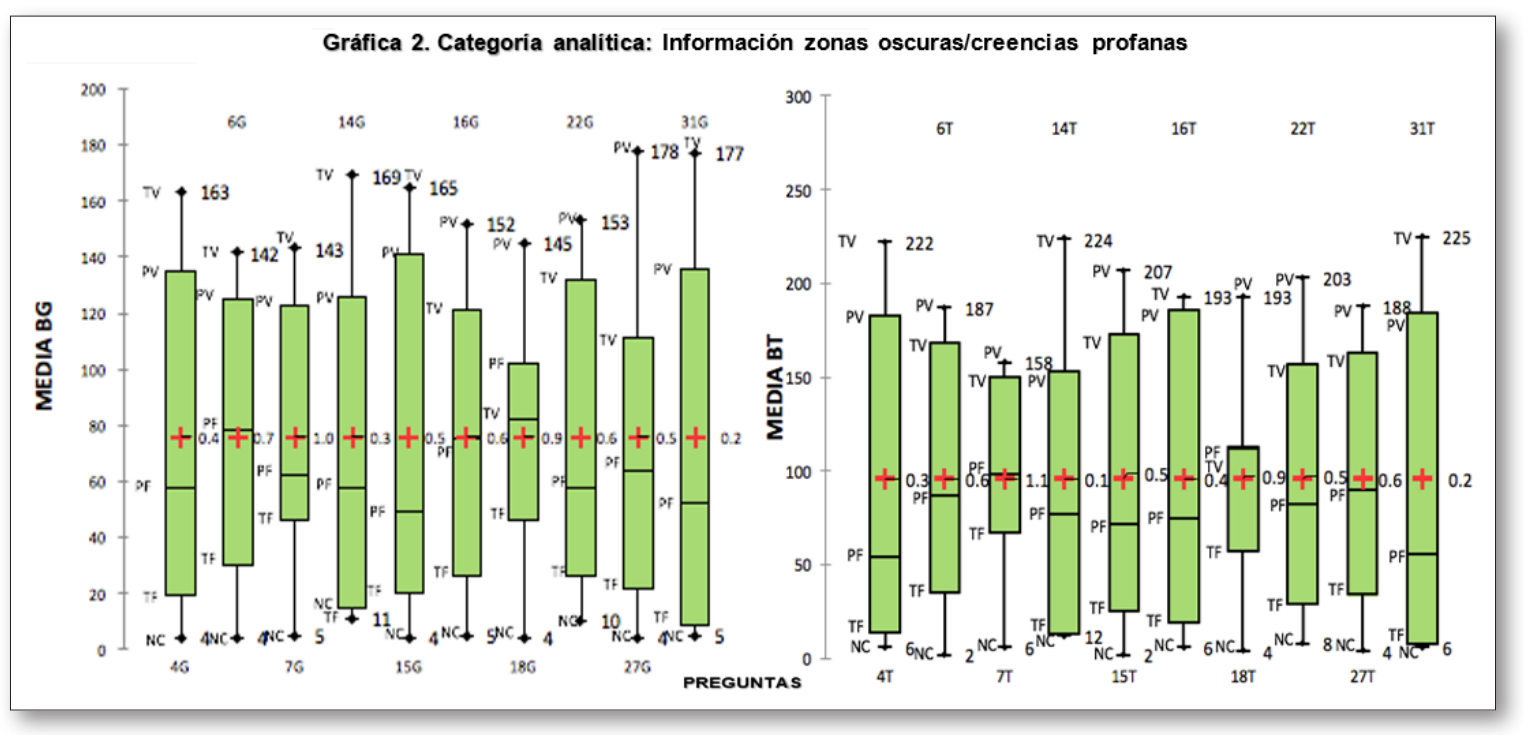

La opción correcta para todas las preguntas es «TF». Como se puede observar en el gráfico 2 y en los datos de medias expuestos en el cuadro 3, en ambos grupos de estudio esta opción registró frecuencia de respuesta baja. En contraste, las opciones de respuesta de mayor frecuencia fueron PV y TV; esto es, 
las opciones opuestas a la respuesta correcta, lo que se refleja en los bajos valores de medias por ítem y por categoría analítica. Es relevante la homogeneidad en las medias para cada pregunta en ambos grupos de estudio, lo que evidencia el manejo de creencias similares en ambos casos. Esto es, un alto porcentaje de estudiantes confía en información incorrecta acerca del CC, como la que establece su relación directa con otros problemas ambientales, de salud o riesgos naturales (terremotos y tsunamis); así como en información difusa, por ejemplo, sobre el hecho de que sus impactos afectarán a toda la población por igual y no de forma diferenciada.

\section{Cuadro 3.}

Valoración de diferencias significativas entre BT y BG en la categoría «Información-Zonas oscuras/creencias profanas»

\begin{tabular}{|c|c|c|c|c|c|}
\hline Pregunta & $\begin{array}{l}M E D I A \\
\quad B G\end{array}$ & $\begin{array}{l}\text { MEDIA } \\
\quad B T\end{array}$ & $\begin{array}{r}\text { MEDIA } \\
\text { General }\end{array}$ & $\begin{array}{c}\text { Valor de } \\
\text { significancia } \\
\text { (TUKEY) }\end{array}$ & $\begin{array}{c}\text { Frecuencia rpta. } \\
\text { (\%) Totalmente } \\
\text { falso }\end{array}$ \\
\hline $\begin{array}{l}\text { 4. El agujero polar del ozono provoca el deshielo } \\
\text { de los polos }\end{array}$ & 0,4 & 0,3 & 0,4 & - & 3,8 \\
\hline $\begin{array}{l}\text { 6. Los cánceres de piel se incrementarán como } \\
\text { resultado del CC }\end{array}$ & 0,7 & 0,6 & 0,6 & 1,0 & 7,6 \\
\hline 7. La lluvia ácida es una de las causas del CC & 1,0 & 1,1 & 1,1 & 0,325 & 13,2 \\
\hline $\begin{array}{l}\text { 14. El efecto invernadero pone en riesgo la vida } \\
\text { en la Tierra }\end{array}$ & 0,3 & 0,1 & 0,3 & 0,982 & 2,8 \\
\hline $\begin{array}{l}\text { 15. El CC aumentará el número de terremotos y } \\
\text { tsunamis }\end{array}$ & 0,5 & 0,5 & 0,5 & 1,0 & 5,2 \\
\hline $\begin{array}{l}\text { 16. El CC es consecuencia del agujero en la capa } \\
\text { de ozono }\end{array}$ & 0,6 & 0,4 & 0,5 & 0,271 & 5,2 \\
\hline $\begin{array}{l}\text { 18. El CC es el resultado de la variabilidad climá- } \\
\text { tica natural }\end{array}$ & 0,9 & 0,9 & 0,9 & 0,936 & 12,0 \\
\hline $\begin{array}{l}\text { 22. La subida de las temperaturas afectará a todas } \\
\text { las regiones del planeta por igual }\end{array}$ & 0,6 & 0,5 & 0,6 & 0,125 & 6,4 \\
\hline $\begin{array}{l}\text { 27. El } \mathrm{CO}_{2} \text { provoca la destrucción de la capa de } \\
\text { ozono }\end{array}$ & 0,5 & 0,6 & 0,5 & 1,0 & 6,5 \\
\hline $\begin{array}{l}\text { 31. El efecto invernadero está ocasionado por la } \\
\text { actividad humana }\end{array}$ & 0,2 & 0,2 & 0,2 & 0,273 & 2,0 \\
\hline Media de la categoría analítica & 0,6 & 0,5 & 0,5 & & \\
\hline
\end{tabular}

El estudio de diferencias significativas entre los dos grupos realizado a través de la prueba ANOVA, se aplicó tomando como factor de cruce la pregunta 4. En el cuadro 2 se muestran los valores de significancia, que revelan que no hay diferencias significativas, ya que todos los valores son superiores a $\mathrm{p}>0,05$. Este dato constata, por una parte, la presencia de información difusa y la prevalencia de creencias profanas acerca del CC en ambos grupos de estudio. Por otra parte, se hace patente el alto peso que esta información tiene en la configuración de la RS acerca del CC para un alto porcentaje de estudiantes, tanto en el BT como en el BG.

Por último, los resultados de la prueba de correlación de Pearson muestran valores altos para las preguntas de esta categoría analítica. De las 45 correlaciones posibles entre los ítems de esta categoría analítica, los resultados arrojan valores que oscilan entre $\mathrm{P}=0,924$ a $\mathrm{P}=0,997$ para 39 de las 45 correlaciones. Para el resto, los valores muestran correlación en el rango de $\mathrm{P}=0,686$ a $\mathrm{P}=0,870$, para la pregunta 18 en relación con las preguntas 4, 7, 14, 15, 17 y 31. Estos datos señalan, además de una 
alta presencia de creencias profanas vinculadas al CC, que dicha información está interrelacionada entre sí. Esto es, revelan la presencia de un campo cognitivo sobre el CC en el que se articulan datos de creencias profanas como en las referidas en las preguntas 6 y 16. Asimismo, indican que dicha articulación de información configura el núcleo central de la representación social acerca del CC para un alto porcentaje de estudiantes de ambos grupos de estudio.

\section{Información-consecuencias}

En esta categoría analítica se exploran los conocimientos acerca de las consecuencias del CC a nivel global y local. El valor Alfa de Cronbach fue de 0.944. En la gráfica 3 se expone la comparación en el estudio estadístico de medias entre los resultados obtenidos por ambos grupos, BG y BT, para cada uno de los ítems de esta categoría analítica. La opción correcta para todos estos es TV. Los valores mínimos hacen referencia al valor «no contestó».

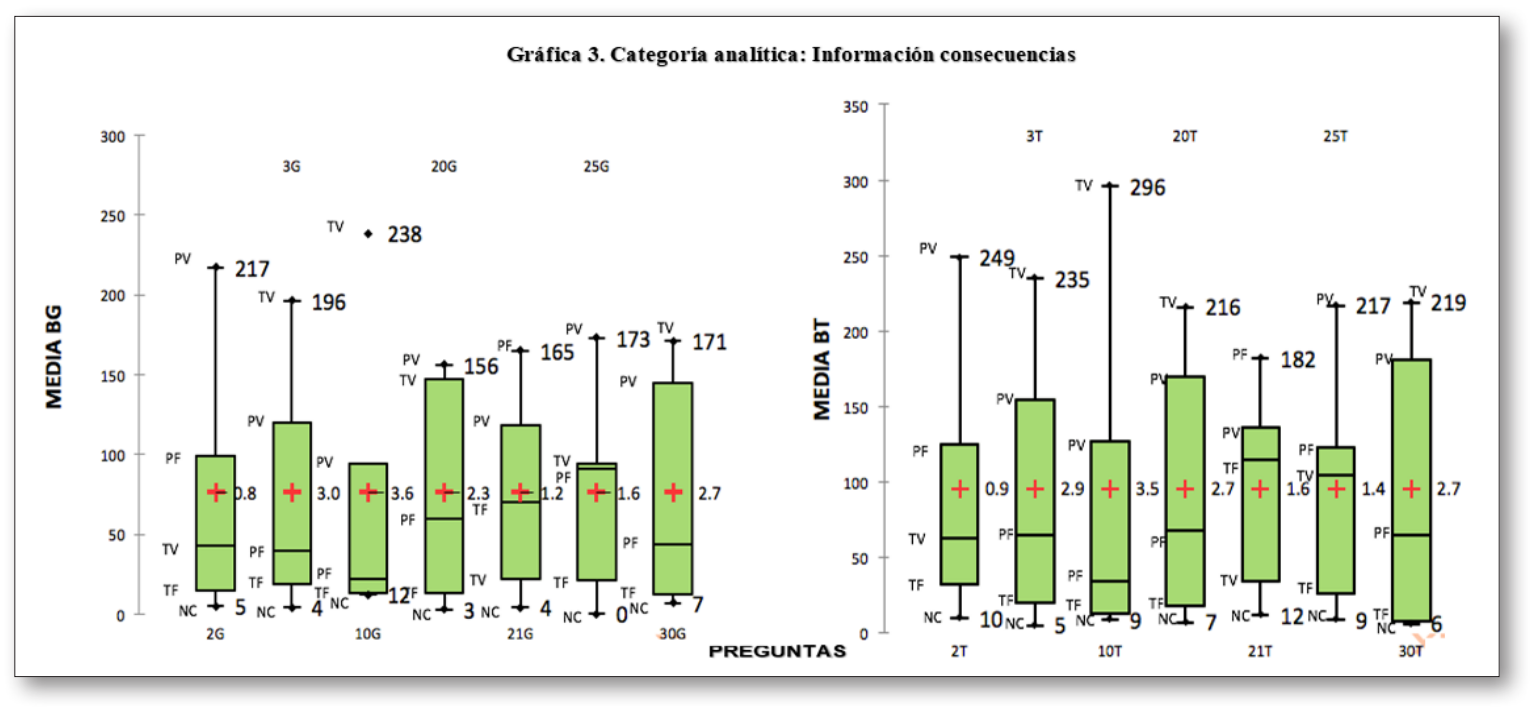

Como se observa, en 4 de los 7 ítems (3, 10, 20 y 30) el valor de las medias supera, para ambos grupos de estudio, el valor 2,5 -que correspondería a $50 \%$ de respuestas correctas-; siendo, para estos casos, la opción de respuesta TV la de mayor frecuencia. Asimismo, la pregunta 21 registró la mayor frecuencia en la opción de respuesta PF, mientras que las preguntas 2 y 25 presentaron la mayor frecuencia en la opción PV. La media para ambos grupos de estudio está en las opciones PF y PV. Esta tendencia en las medias señala que existe homogeneidad en la respuesta, además de mayor puntaje de certeza para ambos grupos de estudio. También es la información acerca de las consecuencias la de mayor presencia en la representación social de los dos grupos, BT y BG, en comparación con la información científica acerca del efecto invernadero y los GEI. Este dato evidencia que la información de procedencia científica está contaminada o reformulada por la cultura común en ambos grupos escolares.

La valoración de diferencias significativas entre ambas submuestras, realizada a través de la prueba ANOVA, ha tomado como factor de cruce la pregunta 2. En el cuadro 4 se muestran los valores de significancia: su lectura revela que no hay diferencias significativas, siendo todos los valores superiores a p $>0,05$. Este resultado puede indicar que la información y los conocimientos acerca de las consecuencias del CC incorporados en el currículo de ambos bachilleratos no tiene influencia significativa en su competencia sobre estas cuestiones. Estos datos confirman que es la información proveniente de la cultura común la que tiene mayor peso frente a la información científica que se puede difundir a 
través del BG y BT en la construcción de la representación social del CC. Asimismo, los datos señalan un mayor conocimiento sobre los impactos del CC a nivel global que sobre los impactos regionales o nacionales.

\section{Cuadro 4.}

Valoración de diferencias significativas entre BT y BG en la categoría «Información-consecuencias»

\begin{tabular}{|l|c|c|c|c|c|}
\hline \multicolumn{1}{|c|}{ Pregunta } & $\begin{array}{c}\text { MEDIA } \\
B G\end{array}$ & $\begin{array}{c}\text { MEDIA } \\
\text { BT }\end{array}$ & $\begin{array}{c}\text { MEDIA } \\
\text { General }\end{array}$ & $\begin{array}{c}\text { Valor de } \\
\text { significancia } \\
\text { (TUKEY) }\end{array}$ & $\begin{array}{c}\text { Frecuencia rpta. } \\
\text { (\%) Totalmente } \\
\text { verdadero }\end{array}$ \\
\hline $\begin{array}{l}\text { 2. Un planeta más cálido ampliará el área de in- } \\
\text { cidencia de las enfermedades tropicales }\end{array}$ & 0,8 & 0,9 & 0,8 & - & 12,4 \\
\hline $\begin{array}{l}\text { 3. El incremento de las temperaturas favorecerá } \\
\text { la ocurrencia de fenómenos atmosféricos extre- } \\
\text { mos (ciclones, huracanes, inundaciones, etc.) }\end{array}$ & 3,0 & 2,9 & 2,9 & 0,709 & 50,2 \\
\hline $\begin{array}{l}\text { 10. Todos los países sufrirán el CC } \\
\text { 20. El nivel del mar está aumentando debido a la } \\
\text { dilatación del agua por el ascenso de la tem- } \\
\text { peraturas }\end{array}$ & 2,3 & 2,7 & 2,5 & 0,419 & 62,2 \\
\hline $\begin{array}{l}\text { 21. El CC disminuirá la cantidad de lluvia en mi } \\
\text { país }\end{array}$ & 1,2 & 1,6 & 1,4 & 0,14 & $6,3,3$ \\
\hline $\begin{array}{l}\text { 25. El CC agudizará los problemas de desertifica- } \\
\text { ción }\end{array}$ & 1,6 & 1,4 & 1,5 & 0,221 & 23,1 \\
\hline $\begin{array}{l}\text { 30. Muchas islas y zonas costeras quedarán su- } \\
\text { mergidas debido al cambio climático }\end{array}$ & 2,7 & 2,7 & 2,7 & 0,148 & 45,5 \\
\hline Media de la categoría analítica & 2,2 & 2,2 & 2,2 & & 6,5 \\
\hline
\end{tabular}

El estudio correlacional mediante la prueba de Pearson sobre estas preguntas revela que de las 21 posibles correlaciones intercategoriales, esta es significativa en cuatro casos con P > 0,05 bilateral: en la pregunta 2 con la $21(\mathrm{P}=0,935)$ y con la $25(\mathrm{P}=0,953)$, lo mismo ocurre con la pregunta 10 , en relación con la $20(\mathrm{P}=0,882)$ y con la $30(\mathrm{P}=0,911)$. Las siguientes preguntas presentan correlación de Pearson significativa en nivel $\mathrm{P}>0,01$ bilateral: la pregunta 3 con la $10(\mathrm{P}=0,970)$, con la $20(\mathrm{P}$ $=0,969)$ y con la $30(\mathrm{P}=0,981)$; la pregunta 20 con la $30(\mathrm{P}=0,997)$ y la 21 con la $25(\mathrm{P}=0,983)$. Por ello es posible inferir que el incremento de la temperatura, la mayor presencia de fenómenos atmosféricos extremos y el aumento en el nivel del mar son los contenidos que los estudiantes reconocen más como consecuencias del CC y, en consecuencia, con mayor nivel de correlación. Destaca que solo existe correlación en dos casos para lo relacionado con el impacto del CC en la salud: con problemas de desertificación y con la alteración del régimen pluvial. Es significativo que no haya correlación con hidrometeoros extremos, pese a que la población de estudio habita en una de las zonas geográficas de mayor impacto por hidrometeoros agudizados por el CC (Tejeda et al., 2012).

\section{Información-causas}

Se exploró la información que maneja la muestra acerca de las causas del CC. El Alfa de Cronbach para este bloque de respuestas fue de 0,323. La gráfica 4 muestra el comparativo de las respuestas en la opción TV, la correcta para las preguntas correspondientes a la categoría. Destaca que en el caso de la pregunta 11, la opción de respuesta TF fue la de mayor frecuencia $(51,7 \%$ para BG y $17,6 \%$ para $\mathrm{BT})$, al contrario de lo que sucede en las preguntas 13 y 17 . Como se observa, el desconocimiento 
de cambios en los patrones de consumo que impactan en el CC es alto. Aunque es relevante que la población de estudio reconoce la causa antrópica del CC y el papel de los combustibles fósiles en su gestación. En cuanto a las causas del fenómeno, los datos revelan que el conocimiento es superficial en ambos grupos, BT y BG, centrado en la influencia antrópica, pero sin profundizar en aspectos más concretos.

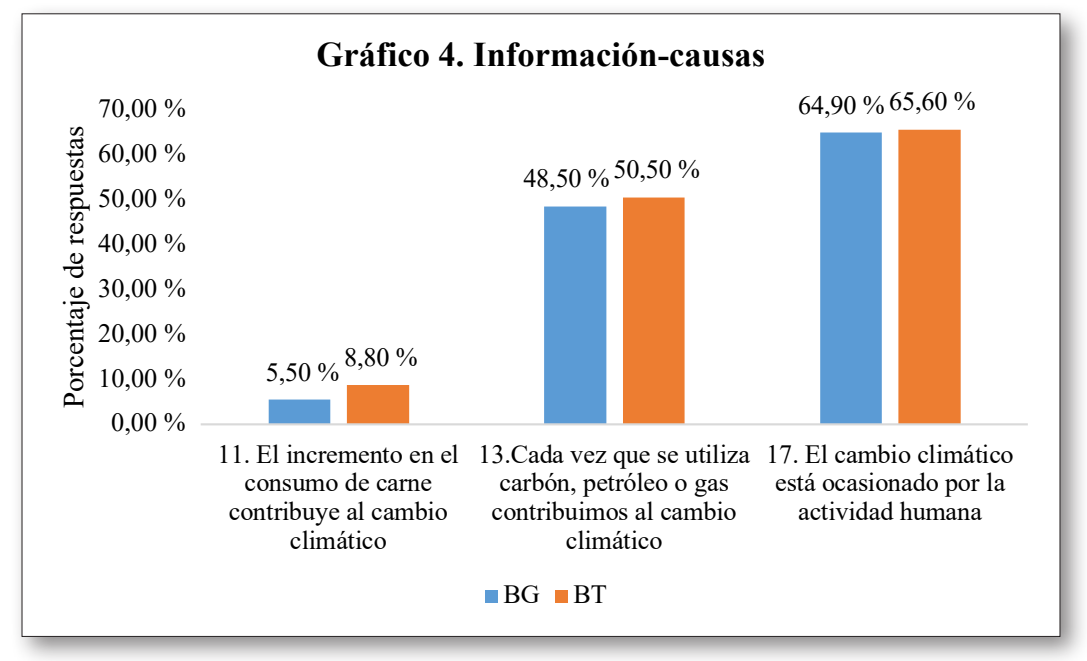

Las diferencias significativas en esta categoría analítica y en las siguientes se valoraron con el estadístico $X^{2}$ debido a su pertinencia por el número de ítems que la conforman (véase el cuadro 5). El grado de significación fue de 0,05 . Es posible inferir que, en este campo de la información, la incidencia del currículo es, en estos bachilleratos, débil. Las correlaciones entre estas tres preguntas también son débiles. Esto es, la pregunta 11 arroja valores de correlación de Pearson con las preguntas 13 de $\mathrm{P}=-0,379$ y $17 \mathrm{P}=0,442$. Estos valores señalan la ausencia de correlación entre el consumo de carnes rojas y el uso de combustibles fósiles como causas del CC. La correlación entre las preguntas 13 y 17 es de P = 0,937, lo que demuestra que los estudiantes asocian la influencia antrópica del CC con el consumo de hidrocarburos (combustibles fósiles), pero no con el de carnes rojas. Este resultado pone nuevamente en evidencia un conocimiento superficial de las causas del CC.

Cuadro 5.

Resultado de $X^{2}$

\begin{tabular}{|c|c|c|}
\hline Categoría analitica & Pregunta: $X^{2}$ & Interpretación \\
\hline Información causas & $11: X^{2}=0,045,13: X^{2}=0,046,17: X^{2}=0,046$ & No hay diferencias significativas (DS) \\
\hline Información sobre CC & $28: X^{2}=0,049,29: X^{2}=0,05$ & No hay DS \\
\hline Acciones & $26: X^{2}=0,049,32: X^{2}=0,053,43: X^{2}=0,16$ & Sí hay DS \\
\hline Representación & $33: X^{2}=0,157,34: X^{2}=0,052,35: X^{2}=0,046$ & Sí hay DS \\
\hline Representación/consecuencias & $38: X^{2}=0,003,39: X^{2}=0,002$ & No hay DS \\
\hline Representación/información & 40: $X^{2}=0,046,41: X^{2}=0,046,42: X^{2}=0,006$ & No hay DS \\
\hline Responsables & $36: X^{2}=0,003,37: X^{2}=0,002$ & No hay DS \\
\hline \multicolumn{3}{|l|}{${ }^{*} \mathbf{X}^{2} \mathrm{p}>0,05, \mathrm{p}>0,01$} \\
\hline
\end{tabular}




\section{Información sobre el CC}

La prueba de confiabilidad realizada mediante el estadístico Alfa de Cronbach para estas preguntas alcanzó un valor de 0,978 . Esta categoría explora los conocimientos científicos relacionados con el CC con base en los más recientes informes del IPCC (2014): sobre la causalidad antrópica del CC actual, así como sobre la historia climática que muestra que se han producido oscilaciones de origen no antrópico. Para ambas respuestas, la opción correcta es TV. De los resultados destaca que, para ambas preguntas, la opción PV fue la de mayor frecuencia (pregunta 28: 42,7 \% en el BG y 46,1 \% en el BT, pregunta 28: 53,3 \% en el BG y 53,2 \% en el BT), siendo la opción correcta la que registra la segunda frecuencia más alta, tal como representa la gráfica 5. Los valores de $X^{2}$ revelan que no hay diferencias significativas para ambos grupos de estudio en estas preguntas, mostrando la misma tendencia que en la categoría acerca de las causas del CC. Asimismo, la correlación de Pearson entre estas dos preguntas es de $\mathrm{P}=0,932$, lo que revela una correlación significativa en el nivel 0,05 (bilateral).

Estos datos en su conjunto evidencian que los estudiantes, tanto de BT como de BG, asocian estos dos asuntos con conocimientos de cultura común en relación con el CC. Sin embargo, las medias (Me) en la pregunta $28, \mathrm{Me}=3,19$, y en la pregunta 29, $\mathrm{Me}=1,93$, señalan que en ambos grupos no hay claridad en cuanto a la diferenciación entre el origen antrópico del CC actual y las oscilaciones climáticas en una escala temporal más amplia. Esta distorsión desemboca en una articulación heterogénea de los datos científicos con los de la cultura común, así como en confusiones conceptuales en relación con el CC y las escalas temporales de la variabilidad climática. Esto es, una débil apropiación de información proveniente de las ciencias del clima.

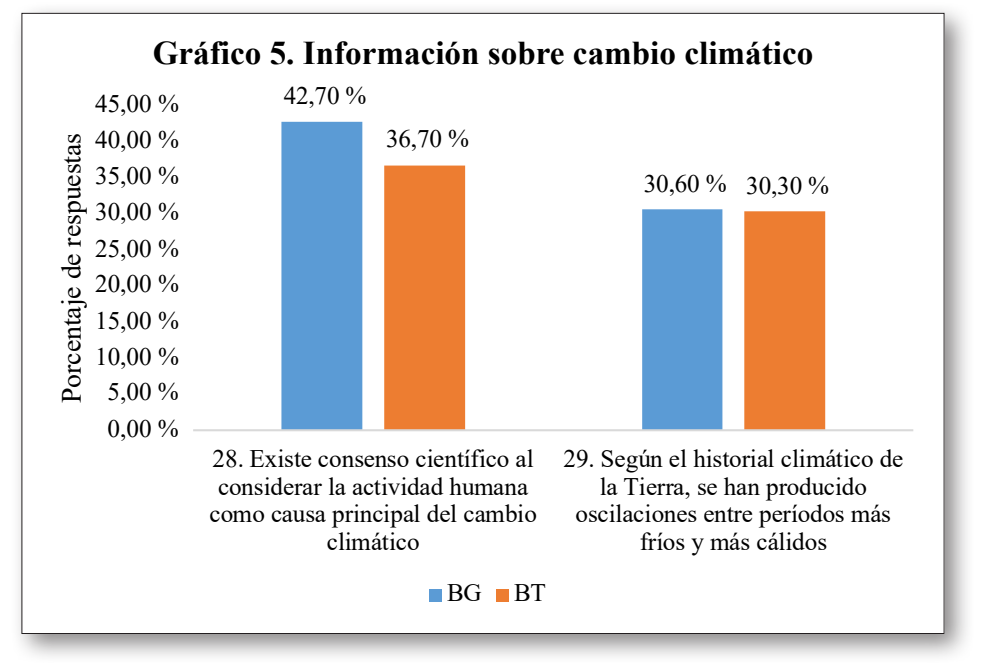

\section{Acciones}

Las acciones de respuesta al CC se exploraron con tres preguntas. Las dos primeras se muestran en la gráfica 6, que indica las frecuencias de respuesta a la opción TV. Se aprecia un porcentaje bajo de respuesta en acciones pertinentes de mitigación. El estadístico $X^{2}$ revela que existen diferencias significativas para la pregunta 32 entre los estudiantes de BG y BT, aunque el consenso frente a estas dos acciones centrales es bajo. El valor de correlación de Pearson para las preguntas 26 y 32 fue de $\mathrm{P}=0,968$, lo que indica que los estudiantes de ambos grupos, BT y BG, no relacionan estas dos acciones de respuesta. Este dato evidencia la ausencia de articulación entre el dominio de conocimientos científicos asociados con el ciclo del carbono y las acciones de mitigación del CC. No obstante, sí se relaciona la emisión de GEI con los modos de transporte. 


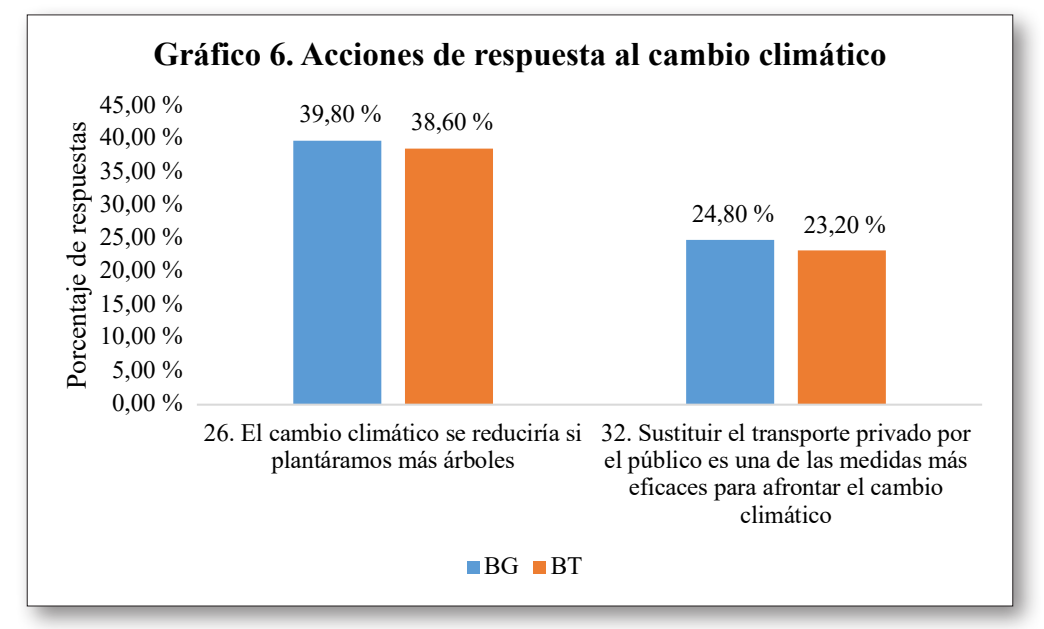

Las acciones escolares desplegadas acerca del CC se valoraron en la pregunta 43. Los resultados revelan que menos del $50 \%$ de los estudiantes reconocen haber participado en alguna experiencia específicamente relacionada con el CC. El valor del estadístico $X^{2}$ señala diferencias significativas entre ambos grupos (BG y BT) al obtener 0,16. Los estudiantes de BT afirman participar en actividades escolares relacionadas con el CC en mayor medida que los estudiantes de BG. Esta diferencia puede deberse a las características curriculares del BT, donde se le otorga mayor relevancia al desarrollo de tareas prácticas afines a las carreras técnicas que se ofertan, vinculadas con actividades relacionadas con la sustentabilidad (SEP, 2017).

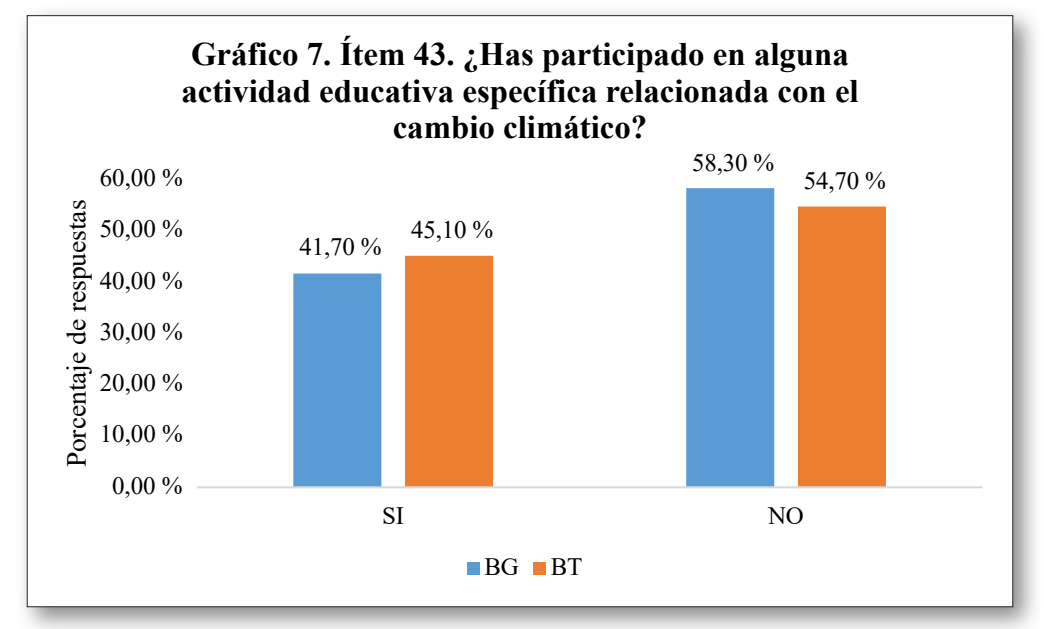

Dada la relevancia de esta pregunta y su correspondencia con la hipótesis de investigación, se analizó su relación con todas las preguntas de la categoría analítica: Información-efecto invernadero/ GEI, a fin de explorar cómo relacionan información procedente de las ciencias del clima con acciones. Se estudió la vinculación entre quienes respondieron TV (respuesta correcta) en este bloque de preguntas y su participación o no en actividades escolares. En la gráfica 8 se exponen los resultados: la línea superior muestra la frecuencia de respuesta correcta a cada ítem (TV); en las líneas inferiores se indican las opciones de respuesta a la pregunta 43 (sí/no han participado en alguna actividad educativa específica relacionada con el CC). Los resultados revelan que los estudiantes que han demostrado un mayor dominio de conocimientos validados científicamente acerca del efecto invernadero y los GEI no muestran una tendencia que relacione este mayor dominio con la realización de acciones escolares 
específicas sobre la cuestión climática. Esto es, aquellos que revelan estar mejor informados sobre estos temas no son, necesariamente, quienes declaran haber participado en más actividades escolares relacionadas con el CC.

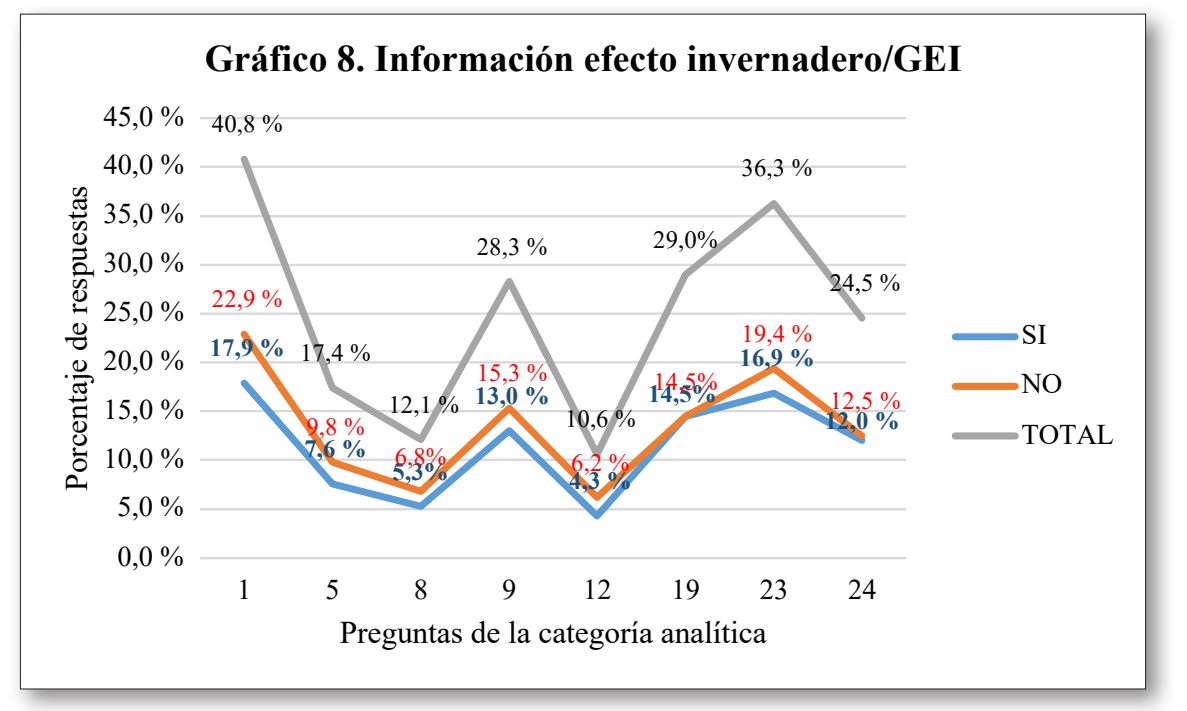

\section{Representación}

El diseño de respuesta en las preguntas que conforman esta categoría analítica, así como el número de preguntas que la conformaron (2), han sido los criterios para determinar la elección de estadístico $X^{2}$ para valorar diferencias. Como se observa en el cuadro 5, únicamente existen estas diferencias en la pregunta 33. Existe consenso casi total en relación con la existencia del CC (98,70 \% en el BG y $97,90 \%$ en el BT) y la pregunta 34, "¿Qué grado de seguridad tienes en la respuesta que has dado a la cuestión anterior?», siendo el $28,5 \%$ de los estudiantes de BG quienes responden estar totalmente seguros y el $25,7 \%$ de BT.

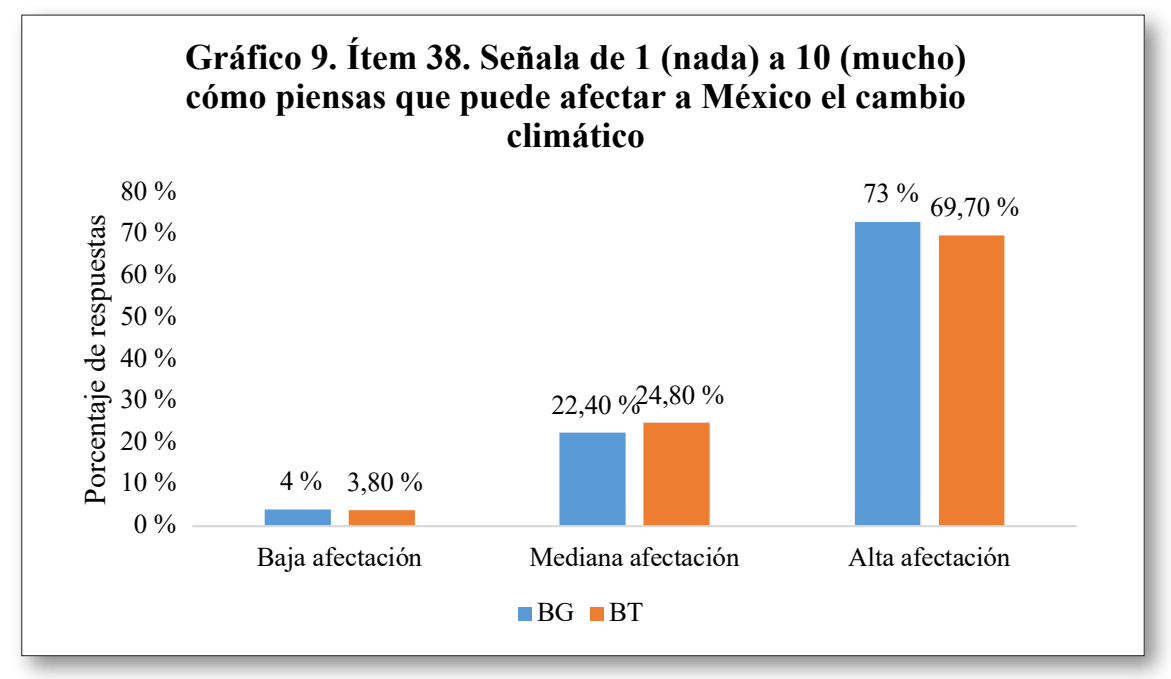

En cuanto al impacto percibido del CC a nivel nacional, los datos revelan que no existen diferencias significativas entre ambos grupos de estudio. Se destaca que pese a ser México un país altamente 
vulnerable a los impactos de CC (IPCC, 2014), no existe un alto consenso en relación con este punto. Los datos se muestran en las gráficas 9 y 10 (preguntas 38 y 39). Esto es, hay consciencia del problema, mas no de las afectaciones y de su potencial de amenaza. Este fenómeno se constata en los valores bajos que muestra la correlación de Pearson entre estas preguntas.

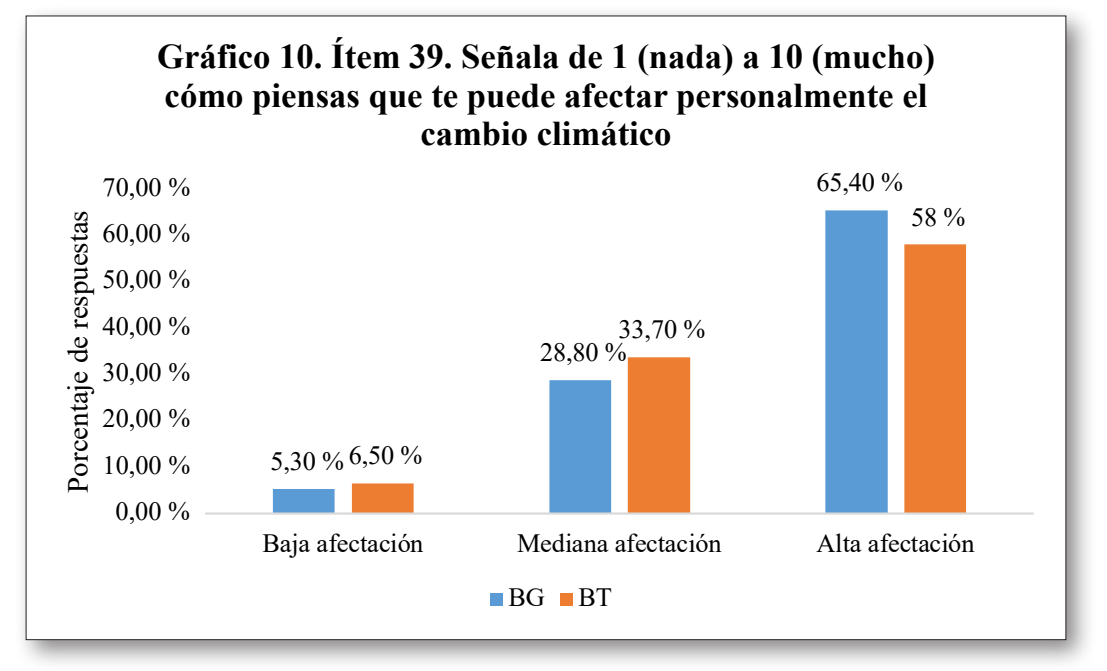

\section{Aportaciones pedagógicas y reflexiones finales}

Los resultados obtenidos en este estudio suscitan diversas reflexiones. En relación con los supuestos hipotéticos que guiaron la investigación las evidencias muestran que la representación del alumnado acerca del CC se está constituyendo tanto con elementos provenientes de la cultura común como de la científica, dando lugar a creencias profanas sobre el fenómeno. En las RS que se están configurando sobre la crisis climática tiene mayor peso la cultura común y las creencias profanas que la articulan, en las que también se incorporan y reconfiguran, según la lógica del sentido común, elementos -conceptos, teorías, imágenes, etc.- provenientes de la representación científica. Los datos más específicos acerca del fenómeno parecen tener poca presencia en el currículo de BT y BG, y, por ende, conllevan una articulación precaria entre causas, consecuencias y acciones a desplegar frente a la crisis climática.

La incidencia del currículo en la representación sobre CC en la población de estudio parece ser débil. La información procedente de la cultura común, con información errónea socialmente construida y con muchas zonas oscuras (Meira, 2011), parece tener mayor peso en la representación que la información procedente del campo científico. Esta realidad se refuerza también por el bajo peso que se le otorga a las experiencias escolares que los estudiantes declaran haber realizado acerca del CC. En el currículo formal siguen pesando tradiciones académicas que relegan el abordaje de estos nuevos temas y problemas, para seguir dando centralidad a los contenidos propios de los campos disciplinares convencionales, lo que sucede tanto en el BG como en el BT. En líneas generales, se puede afirmar que el CC está más presente en el currículum de los bachilleratos más como tema-como contenidos dispersos y poco articulados en las diferentes disciplinas- que como problema -incorporando y articulando las diferentes dimensiones de la crisis climática, desde las científico-naturales hasta las éticas, económicas y socio-culturales-.

En cuanto al nivel de información acerca del CC, los resultados en ambos bachilleratos evidencian que la formación tiende a limitarse a tópicos generales sobre el fenómeno. En aspectos de mayor especificidad sobre los GEI, sobre su origen y sobre su incidencia en los impactos del CC la precariedad de la representación es evidente, aunque se verifique un reconocimiento generalizado de la responsabilidad 
antrópica en el fenómeno. Este dato coincide con estudios similares que exploran la dimensión social del CC (Breslyn, Drewes, McGinnis, Hestness, E. y Mouza, 2017; Collos e Ison, 2009; Feinber y Willwe, 2010; Hestness, McGinnis y Breslyn, 2016). Destaca la simetría en la representación del CC entre ambos grupos de estudio, con escasas diferencias significativas. También, algunas diferencias en las acciones a desplegar más que en el campo de la información, con mayor presencia en el grupo de BT.

Asimismo, destaca la información obtenida a partir del análisis de las correlaciones entre las categorías analíticas estudiadas. Llama la atención la configuración de los campos cognitivos (Bigge y Hunt, 1979) acerca del CC. Los que presentan menos correlaciones son los relacionados con la información científica acerca de los GEI y el efecto invernadero, y con la identificación de las causas y las consecuencias. En contraparte, los que tienen mayor correlación son los asociados con el vínculo científicamente erróneo entre el CC y diversos problemas ambientales (agujero de la capa de ozono, lluvia ácida).

Estos datos revelan que la representación del CC en la muestra del estudio se configura más con información construida socialmente en el marco de la cultura común y con información proveniente de medios de comunicación e internet, más que de experiencias escolares que trasponen información científica sobre el CC. Es relevante también la presencia en la representación de la influencia antrópica y de su relación con algunas acciones de mitigación y adaptación. Aunque esto es un avance, es necesario trabajar en darle mayor articulación y abordar un abanico más amplio de conocimientos sobre las acciones necesarias para enfrentar los impactos del CC, principalmente desde la perspectiva de la adaptación, pero también de la mitigación.

Es necesario repensar cómo se incorpora el CC en el currículo del BG y BT, por una parte, y por otra cómo se desarrolla en las diversas tareas educativas este -en la praxis curricular-. Esto es, de qué manera se explicita en el currículo formal y cómo es mediado a través de las prácticas docentes en el currículo vivido (Valladares, 2017). Pese a los avances en cuanto a incorporación de enfoque de CTSA, los problemas ambientales, entre ellos el CC, continúan ocupando espacios periféricos, con una visión fragmentada y reduccionista, limitada las más de las veces al estudio de su dimensión biofísica, sin considerar sus dimensiones social y política, que merecen ser estudiadas y discutidas en los espacios académicos dado que son y serán claves para la transición socioecológica que permita enfrentar los retos de la crisis climática con ciertas garantías de éxito a medio y largo plazo. Es necesario situar la problematización de lo ambiental como elemento aglutinador, tanto de los contenidos como de las actividades escolares. Esto es, propiciar de manera articulada lo relacionado con conocer-saber con saber-hacer y también con saber-ser para el cambio socioambiental (Sauvé, 2014).

El CC tiene que abordarse desde visiones complejas que consideren integralmente sus dimensiones social, histórica y natural, y que posibiliten conectar los conocimientos con las acciones de respuesta al CC conectando diferentes escalas - de lo local a lo global-. Para ello, propuestas pedagógicas como la de Dewey, acerca de la vinculación entre saber y hacer (Giolitto, 1984), la de Sauvé (2014), sobre la formación de competencias ecociudadanas, la del aprendizaje social (Collos e Ison, 2009; De Souza, 2004) o la resiliencia social ante el CC (Cacioppo, Reis y Zautra, 2011; Cheshire, Esparcia y Shucksmith, 2015; Folke, 2006; Kais e Islam, 2016; Keck y Sakdapolrak, 2013) pueden resultar pertinentes para formular rutas pedagógicas y didácticas sobre y para el CC que permitan el despliegue de acciones colectivas que sean efectivas.

Esto es, la gestión de aprendizajes que no se centren solo en contenidos relacionados con la alfabetización climática. La pedagogía para la resiliencia frente al CC ha de tener como horizonte la construcción colectiva de un mundo más armónico en términos de la relación entre el ser humano y el medio ambiente, donde se reconozca su ecodependencia, que incorpore asuntos éticos y sociales como la «justicia climática» (Mendoza, 2009), formas responsables de consumo de recursos y la resiliencia social para enfrentar los impactos y las causas del CC, entre otras dimensiones. Conocer para aprender a entrar en acción frente a asuntos socioambientales. 
Es preciso incorporar estrategias de aprendizaje social en las actividades académicas (De Souza, 2004). Estrategias que se construyan en la interacción con el medio ambiente y que vinculen los conocimientos científicos con el saber-hacer individual y colectivo, a través de marcos éticos de respeto y cuidado del medio ambiente, y para generar competencias ecociudadanas. Asimismo, es necesario que se reconozca la relevancia social y política de estos aprendizajes y de la función cultural imprescindible de los centros escolares en las respuestas de mitigación y adaptación al CC, así como la articulación de estos aprendizajes con los enfoques de resiliencia social, entendida como la capacidad que desarrolla un colectivo social para enfrentar perturbaciones y/o nuevas condiciones de vida y dinamizar acciones comunitarias de gestión con los diversos agentes sociales involucrados. Estos enfoques requieren el desarrollo y fortalecimiento de redes inter e intracomunitarias, con el propósito de enfrentar eventos extremos en el futuro y/o cambios en sus condiciones de vida asociados a las condiciones de CC (Cheshire et al., 2015; Kais e Isam, 2016; Keck y Sakdapolrak, 2013). En este marco de recomendaciones, la formación ecociudadana en el Bachillerato es de central importancia, al menos, por dos razones: la etapa crítica en la que se encuentran los y las estudiantes que la cursan (alcanzan su madurez cognitiva y moral) y la alta probabilidad para muchos de ellos y ellas de que sea esta la última oportunidad para conectar con la amenaza climática dentro del sistema educativo.

El CC es el gran desafío para la humanidad. No únicamente por su nivel de complejidad científica, sino por su alta relevancia social, política y económica, que manifiestan una urgencia en la gestión y el despliegue de acciones de respuesta, urgencia que también debe ser apremiante para el sistema educativo.

\section{BIBLIOGRAFÍA}

Allen, L. B y Crowley, K. (2017). Moving beyond scientific knowledge: leveragin participation, relevance, and interconnectedness for climate education. International Journal of Global Warming, 12(3/4), 299-312.

https://doi.org/10.1504/IJGW.2017.10005878

Bangay, C. y Blum, N. (2010) Education Responses to Climate Change and Quality: Two Parts of the Same Agenda? International Journal of Educational Development, 30(4), 335-450. https://doi.org/10.1016/j.ijedudev.2009.11.011

Bello, B. L. (2017). Las representaciones sociales sobre cambio climático de estudiantes de bachillerato tecnológico. El caso de dos escuelas del estado de Veracruz (tesis de doctorado). Xalapa, México: IIE-UV.

Bello, B. L., Meira, C. P. A. y González, G. E. J. (2017). Representaciones sociales sobre cambio climático en dos grupos de estudiantes de educación secundaria de España y bachillerato de México. Revista Mexicana de Investigación Educativa, 22(73), 505-532. Obtenido de https://www.redalyc. org/pdf/140/14050493008.pdf

Bigge, M. L. y Hunt, M. P. (1979). Bases psicológicas de la educación. CDMX, México: Trillas.

Breslyn, W., Drewes, A., McGinnis, R., Hestness, E. y Mouza, C. (2017). Development of an Empirically-Based Conditional Learning Progression for Climate Change. Science Education International, 28(3), 214-223. Obtenido de http://sustainability.psu.edu/fieldguide/resources/breslyn-w-drewesa-mcginnis-j-r-hestness-e-mouza-c-n-d-development-of-an-empirically-based-conditional-learning-progression-for-climate-change/

Cacioppo, J., Reis, H. y Zautra, A. (2011). Social Resilience. The Value of Social Fitness with an Application to the Military. American Psychologist, 66(1), 43-51.

https://doi.org/10.1037/a0021419 
Carr, W. (1995). Una teoría para la educación. Hacia una investigación educativa crítica. Madrid, España: Morata.

Cheshire, L., Esparcia, J. y Shucksmith, M. (2015). Community resilience, social capital and territorial governance. Revista de Estudios sobre Población y Desarrollo Rural, 18(enero-julio), 7-38. https:// doi.org/10.4422/ager.2015.08.

Collos, K. e Ison, R. (2009). Jumping off Arnstein's Ladder: Social Learning as a New Policy Paradigm for Climate Change Adaptation. Environmental Policy and Governance, 19, 358-373.

https://doi.org/10.1002/eet.523

De Souza Santos, B. (2004). La educación latinoamericana en el siglo XXI. Escenarios hacia las pedagogias de la alienación, domesticación y transformación. San José de Costa Rica, Costa Rica: Instituto Internacional de Investigación sobre Políticas Alimentarias (IFPRI). Obtenido de http://www.migrante. gob.ec/wp-content/uploads/downloads/2012/07/Souza-Educacion_Latinoamericana_en_el_SigloXXI.pdf

Fernández, I. M, Pires, D. M. y Villamañán, R. M. (2014). Educación científica con enfoque cienciatecnología-sociedad-ambiente: construcción de un instrumento de análisis de las directrices curriculares. Formación Universitaria, 7(5), 23-32.

http://dx.doi.org/10.4067/S0718-50062014000500004.

Folke, C. (2006). Resilience: The emergence of a perspective for social-ecological systems analyses. Global Environmental Change, 16, 253-267.

Giolitto, P. (1984). Pedagogía del medio ambiente. Barcelona: Herder.

González G. E. J. y Maldonado González, A. L. (2013) Los jóvenes universitarios y el cambio climático: un estudio de representaciones sociales. Xalapa, México: Editora de la Universidad Veracruzana.

Hestness, J. H., McGinnis, R. y Breslyn, W. (2016). Examining the relationship between middle school students' sociocultural participation and their ideas about climate change. Environmental Education Research

http://dx.doi.org/10.1080/13504622.2016.1266303

Ibáńez, T. (1994). Psicología Social Construccionista. Ciudad de México: Universidad de Guadalajara.

IPCC (2014). Cambio climático. Impactos, adaptación y vulnerabilidad. Resumen para responsables de politicas. Contribución del Grupo de trabajo II al Quinto Informe de Evaluación del IPCC. Suiza: OMM, PNUMA.

Jodelet, D. (2008). La representación social: fenómeno, concepto y teoría. En S. Moscovici (Coord.), Psicología social II. Pensamiento y vida social. Psicología social y problemas sociales (pp. 469-494). Madrid: Paidós.

Kais, S. e Islam, M. (2016). Community Capitals as Community Resilience to Climate Change: Conceptual Connections. International Journal of Environmental Research and Public Health, 13(12), 1211. MDPI AG.

http://dx.doi.org/10.3390/ijerph13121211.

Keck, M. y Sakdapolrak, P. (2013). What is social resilience? Lessons learned and ways. Erdkunde, 67(1), 5-19.

http://dx.doi.org/10.3112/erdkunde.2013.01.02

Lorenzoni, I. y Pidgeon, N. (2005) Defining dangers of climate change and individual behaviour: Closing the gap. Avoiding Dangerous Climate Change Conference, Exeter, UK, February 2005. http://dx.doi.org/10.1177/1075547008329201

Meira, C. P. A. (2011). La sociedad ante el cambio climático. Conocimientos, valoraciones y comportamientos en la población española. Madrid: Fundación Mapfre. 
Meira, C. P. A., Gutiérrez-Pérez, J., Arto-Blanco, M. y Escoz-Roldán, A. (2018). Influence of academic education vs. common culture on the climate literacy of university students / Formación académica frente a cultura común en la alfabetización climática de estudiantes universitarios. Psyecology. http://dx.doi.org/10.1080/21711976.2018.1483569

Mendoza, M. (2009). Justicia Climática: Una tarea pendiente. Managua: Centro de Estudios Internacionales.

Moscovici, S. (1979). El psicoanálisis, su imagen y su público. Buenos Aires: Huemal.

OECD (2009). Green at Fifteen? How 15-year-olds perform in environmental science and geoscience in PISA. París: OECD.

O’Neill, S. J. y Hulme, M. (2009). An iconic approach for representing climate change. Global Environmental Change, 19(4), 402-410.

https://doi.org/10.1016/j.gloenvcha.2009.07.004

Sauvé, L. (2014). Educación ambiental y ecociudadanía. Dimensiones clave de un proyecto políticopedagógico. Revista Cientifica, 18(1), 12-23. https://doi.org/10.14483/23448350.5558

SEP (2017). Planes de estudio de referencia del Marco Curricular Común de la Educación Media Superior. Ciudad de México: Subsecretaría de Educación Media Superior.

Stapleton, M. K. y Sezen-Barrie, A. (2017). A scientist, teacher educator and teacher collaborative: Innovative professional learning design focused on climate change and lessons learned from $\mathrm{K}-12$ classrooms. Innovations in Science Teacher Education, 2(4). Obtenido de http://innovations.theaste. org/a-scientist-teacher-educator-and-teacher-collaborative-innovativeprofessional-learning-designfocused-on-climate-change-and-lessons-learned-from-k-12-classrooms/

Stevenson, R., Nicholls, J. y Whitehouse, H. (2017). What is climate change education? Curriculum Perspectives, 37(1), 67-71. https://doi.org/10.1007/s41297-017-0015-9

Tejeda-Martínez, A., Montes, C. E. y Sarabia, B. C. (2012). Las Inundaciones de 2010 en Veracruz. Vulnerabilidad y adaptación. Xalapa, México: Consejo Veracruzano de Investigación Científica y Desarrollo Tecnológico.

Wagner, W., Hayes, N. F. y Flores Palacios (EDs.). (2011). El discurso de lo cotidiano y el sentido común: La teoría de las representaciones sociales. México-Barcelona: UNAM-Anthrophos.

Valladares, L. (2017). La "práctica educativa» y su relevancia como unidad de análisis ontológico, epistemológico y sociohistórico en el campo de la educación y la Pedagogía. Perfiles Educativos, XXXIX(158), 186-203. Obtenido de http://www.scielo.org.mx/scielo.php?script=sci_arttext\&pid $=$ S0185-26982017000400186 


\section{Climate change in high school. Pedagogical contributions to its approach}

\author{
Laura O. Bello Benavides \\ Instituto de Investigaciones \\ en Educación, Universidad \\ Veracruzana, México \\ labello@uv.mx
}

\author{
Gloria E. Cruz Sánchez \\ Instituto de Investigaciones \\ en Educación, Universidad \\ Veracruzana, México \\ gcruz@uv.mx
}

\author{
Pablo Á. Meira Cartea \\ Faculta de Ciencias da \\ Educación, Departamento \\ de Pedagoxía e Didáctica, \\ Universidad de Santiago \\ de Compostela, España \\ pablo.meira@usc.es
}

\author{
Édgar J. González Gaudiano \\ Instituto de Investigaciones \\ en Educación, Universidad \\ Veracruzana, México \\ edgagonzalez@uv.mx
}

CC reaches the $21^{\text {st }}$ century becoming the most complex socio-environmental problem. This research is part of the field of environmental education and educational research about the social dimension of CC. It is part of an international educational research project, RESCLIMA, which focuses its attention on how secondary and high school students, aged 15 to 17 , take CC science -through the curriculum and other forms of dissemination and propagation of scientific representations- and integrate it in their SRs about the phenomenon. The study was conducted with samples of teenagers from five countries: Brazil, Spain, Italy, Mexico and Portugal during 2017 and 2018. The sample that corresponds to Mexico, which is the one presented here, was conducted in the state of Veracruz, in schools of BG and BT, whose purpose, in addition to contributing to the aforementioned international project, was to provide empirical elements to formulate educational strategies that articulate the study programs in force at this educational level, so as to contribute to generating eco-citizen attitudes to the climate crisis.

The assumptions that have guided the study are: 1) that the constitution of SRs on CC is mediated by the integration of common and scientific culture, although they tend to be at the center of non-scientific theories; and 2) that the differentiated curriculum of the BT and BG could influence the representation generating some differences in the SRs between the two study groups.

The methodological route was quantitative. A questionnaire with 43 closed questions was used: 32 items are Likert type (four answer options), and the rest are closed (varied number of answer options). The Mexican study population consisted of a total of 858 BT and BG students. Seven analytical categories were raised, taking into account the SRs, the causes, the consequences and the concept of CC itself.

The analytical treatment of the data was statistical, previously collected and coded in the SPSS software. The first part consisted of the elaboration of descriptive data of the SRs. Secondly, the tests were performed: Cronbach's alpha to assess reliability. Significant differences between the two study groups were determined by one-way analysis of variance (ANOVA) and with the X2 statistic, together with a Tukey post hoc test. The correlation between items was assessed with Pearson's Correlation.

The results obtained in this study give rise to various reflections. In relation to the hypothetical assumptions, the evidence shows that the students' SRs about CC are constituted with both elements from the common and scientific culture, giving rise to profane beliefs about the phenomenon. The common culture and the profane beliefs that articulate it have a greater impact, in that they are also incorporated and reconfigured, according to the logic of common sense, elements - concepts, theories, images, etc.- from the scientific representation. The more specific data about the phenomenon seem to have little presence in the BT and BG curriculum, and, therefore, entail a precarious articulation between causes, consequences and actions to be deployed in the face of the climate crisis.

The incidence of the curriculum in the representation of CC in the study population seems to be weak. This reality is also reinforced by the low weight given to school experiences that students declare to have done about CC. In general, it can be said that CC is more present in the curriculum of high school students more as a subject -as dispersed and poorly articulated content in the different disciplines- than as a problem -incorporating and articulating the different dimensions of the climate crisis, from the natural scientists to the ethical, economic and socio-cultural-. 